\title{
Antimicrobial susceptibility of Pasteurella multocida and Haemophilus parasuis isolates associated with porcine pneumonia
}

\author{
Kateřina Nedbalcová, Zdenka Kučerová \\ Veterinary Research Institute, Brno, Czech Republic \\ Received May 17, 2012 \\ Accepted October 23, 2012
}

\begin{abstract}
Pasteurella multocida and Haemophilus parasuis pig isolates obtained in the Czech Republic were tested for their susceptibility against selected antimicrobial agents by broth microdilution method between 2008 and 2011. A low degree of resistance was observed for ampicillin, amoxicillin/clavulanic acid, ceftiofur, tulathromycin, tilmicosin, florfenicol and enrofloxacin in $20(6.0 \%), 15(4.5 \%), 2(0.6 \%), 8(2.4 \%), 13(3.9 \%), 5(1.5 \%)$ and $5(1.5 \%)$ P. multocida isolates as well as for tiamulin, gentamicin, tulathromycin, tilmicosin and ampicillin in $2(2.4 \%)$, $2(2.4 \%), 3(3.6 \%), 3(3.6 \%)$ and $6(7.2 \%) \mathrm{H}$. parasuis isolates. In addition, moderate level of resistance to tiamulin was found in $60(18.1 \%) P$. multocida isolates and high level of resistance for tetracycline was detected in $107(32.2 \%)$ P. multocida isolates and in $23(27.7 \%) \mathrm{H}$. parasuis isolates. Differences between resistance rates of $P$. multocida and $H$. parasuis were significant $(P \leq 0.5)$ only for tiamulin. These data confirmed that antimicrobial resistance is not very widespread among current porcine $P$. multocida and $H$. parasuis isolates in the Czech Republic.
\end{abstract}

Antimicrobial resistance, minimal inhibition concentration, respiratory diseases, pigs

Pasteurella multocida and Haemophilus parasuis are the causative agents of infectious diseases of porcine respiratory tract which cause major economic losses by their negative impact on the weight gain, feed conversion, and health status (Oliveira and Pijoan 2004; Shin et al. 2005). Antibiotic treatment is one of the commonly used measures for the control of pasteurellosis and $H$. parasuis infections but the use of antimicrobial agents leads to both selection and increase of resistance (Schwarz and Chaslus-Dancla 2001). Failure to treat infectious disease caused by resistant bacteria leads to increased morbidity and mortality (Kolar et al. 2010). Variations in the antimicrobial use for the control of bacterial pathogens in pigs from one country to another can contribute to evident differences in antimicrobial susceptibility of $P$. multocida and $H$. parasuis. In accordance with the recommendation of Schwarz et al. (2010) on the requirement of application of the same methodology and interpretive criteria (which would allow for re-evaluation of the original data if the interpretive criteria change over time), only a limited number of recent studies were conducted regarding the susceptibility of $P$. multocida and $H$. parasuis to antimicrobials with the use of microdilution method.

Considering the lack of recent studies of antimicrobial susceptibility testing of P. multocida and $H$. parasuis within the European territory, the aim of this work was to examine a panel of various isolates obtained from different Czech locations to determine their susceptibility to selected antimicrobial agents by the broth microdilution method, in accordance with the guidelines issued by the Clinical and Laboratory Standards Institute (CLSI) in 2008.

\section{Materials and Methods}

Sampling

All $P$. multocida and $H$. parasuis isolates were obtained from the lungs of growing pigs that died due to acute respiratory diseases. No more than one isolate of $P$. multocida or $H$. parasuis from the same farm per a six-month period was included in the study. Isolates from animals that had been treated with antimicrobials during two weeks prior to sampling were not included in this study.

Address for correspondence:

Kateřina Nedbalcová

Veterinary Research Institute, Hudcova 70

62132 Brno, Czech Republic
Phone: +420 533331217
Fax: +420 541211229
E-mail: nedbalcova@vri.cz
http://actavet.vfu.cz/ 
Table 1. Comparison of minimal inhibitory concentration of antibiotic between Pasteurella multocida and Haemophilus parasuis isolates from the Czech Republic

\begin{tabular}{|c|c|c|c|c|c|c|c|c|c|c|c|c|c|c|c|}
\hline \multirow{2}{*}{$\begin{array}{l}\text { Antibiotic } \\
\text { and bacterial } \\
\text { species }\end{array}$} & \multicolumn{15}{|c|}{ Number of isolates with MIC (mg/l) } \\
\hline & 0.12 & 0.25 & 0.5 & 1 & 2 & 4 & 8 & 16 & 32 & 64 & 128 & 256 & $\mathrm{MIC}_{50}$ & $\mathrm{MIC}_{90}$ & $\begin{array}{l}\% \\
\mathrm{R}\end{array}$ \\
\hline \multicolumn{16}{|l|}{ Ampicillin } \\
\hline P. multocida & & & 307 & 5 & 2 & 4 & 2 & 1 & 1 & 2 & 8 & & $\leq 0.5$ & $\leq 0.5$ & 6.0 \\
\hline H. parasuis & & & 72 & 5 & 2 & 1 & 0 & 1 & 1 & 0 & 1 & & $\leq 0.5$ & 1 & 7.2 \\
\hline \multicolumn{16}{|l|}{$\begin{array}{l}\text { Amoxicillin } \\
\text { clavulanic acida }\end{array}$} \\
\hline P. multocida & & & 312 & 5 & 1 & 5 & 2 & 3 & 0 & 2 & 2 & & $\leq 0.5$ & $\leq 0.5$ & 4.5 \\
\hline H. parasuis & & & 82 & 1 & 0 & 0 & 0 & 0 & 0 & 0 & & & $\leq 0.5$ & $\leq 0.5$ & 0 \\
\hline \multicolumn{16}{|l|}{ Ceftiofur } \\
\hline P. multocida & & & 327 & 2 & 1 & 0 & 0 & 0 & 0 & 1 & 1 & & $\leq 0.5$ & $\leq 0.5$ & 0.6 \\
\hline H. parasuis & & & 79 & 3 & 0 & 1 & 0 & 0 & 0 & 0 & & & $\leq 0.5$ & $\leq 0.5$ & 0 \\
\hline \multicolumn{16}{|l|}{ Tulathromycin } \\
\hline P. multocida & & & 7 & 86 & 152 & 64 & 12 & 3 & 0 & 6 & 2 & & 2 & 4 & 2.4 \\
\hline H. parasuis & & & 9 & 34 & 20 & 8 & 6 & 3 & 0 & 3 & & & 1 & 8 & 3.6 \\
\hline \multicolumn{16}{|l|}{ Tilmicosin } \\
\hline P. multocida & & & & 41 & 93 & 88 & 84 & 13 & 2 & 3 & 1 & 7 & 4 & 8 & 3.9 \\
\hline H. parasuis & & & & 36 & 26 & 10 & 8 & 0 & 1 & 0 & 0 & 2 & 2 & 8 & 3.6 \\
\hline \multicolumn{16}{|l|}{ Florfenicol } \\
\hline P. multocida & & 84 & 223 & 11 & 2 & 7 & 4 & 0 & 0 & 1 & & & 0.5 & 0.5 & 1.5 \\
\hline H. parasuis & & 49 & 20 & 4 & 8 & 2 & 0 & 0 & 0 & & & & $\leq 0.25$ & 2 & 0 \\
\hline \multicolumn{16}{|l|}{ Flumequine } \\
\hline P. multocida & & & 305 & 5 & 5 & 4 & 5 & 3 & 3 & 2 & & & $\leq 0.5$ & $\leq 0.5$ & \\
\hline H. parasuis & & & 49 & 11 & 17 & 5 & 0 & 1 & 0 & 0 & & & $\leq 0.5$ & 2 & \\
\hline \multicolumn{16}{|l|}{ Enrofloxacin } \\
\hline P. multocida & 320 & 3 & 4 & 4 & 1 & 0 & 0 & 0 & & & & & $\leq 0.12$ & $\leq 0.12$ & 1.5 \\
\hline H. parasuis & 81 & 1 & 1 & 0 & 0 & 0 & 0 & 0 & & & & & $\leq 0.12$ & $\leq 0.12$ & 0 \\
\hline \multicolumn{16}{|l|}{ Tetracycline } \\
\hline P. multocida & & & 201 & 24 & 12 & 15 & 17 & 27 & 23 & 7 & 6 & & $\leq 0.5$ & 32 & 32.2 \\
\hline H. parasuis & & & 56 & 4 & 6 & 2 & 6 & 5 & 2 & 2 & & & $\leq 0.5$ & 16 & 27.7 \\
\hline \multicolumn{16}{|l|}{ Tiamulin } \\
\hline P. multocida & & & 0 & 0 & 9 & 20 & 78 & 165 & 48 & 3 & 9 & & 16 & 32 & 18.1 \\
\hline H. parasuis & & & 7 & 12 & 16 & 29 & 15 & 2 & 2 & 0 & & & 4 & 8 & 2.4 \\
\hline \multicolumn{16}{|l|}{ Gentamicin } \\
\hline P. multocida & & & 13 & 25 & 124 & 129 & 37 & 4 & 0 & 0 & & & 4 & 8 & 1.2 \\
\hline H. parasuis & & & 20 & 12 & 23 & 22 & 4 & 2 & 0 & 0 & & & 2 & 4 & 2.4 \\
\hline \multicolumn{16}{|l|}{$\begin{array}{l}\text { Trimethoprim } \\
\text { sulfamethoxazole }\end{array}$} \\
\hline P. multocida & & 224 & 20 & 22 & 19 & 10 & 12 & 3 & 13 & 9 & & & $\leq 0.25$ & 8 & \\
\hline H. parasuis & & 54 & 7 & 11 & 4 & 4 & 1 & 1 & 0 & 1 & & & $\leq 0.25$ & 2 & \\
\hline
\end{tabular}

${ }^{a}$ Amoxicillin and clavulanic acid in the ratio 2:1; test ranges are expressed as the amoxicillin concentration.

${ }^{\mathrm{b}}$ Trimethoprim and sulfamethoxazole in the ratio 1:19; test ranges are expressed as the trimethoprim concentration.

The dilution ranges tested are those contained within in white area. Values above this range indicate MIC values higher than the highest concentration in the range. Values corresponding to the lowest concentration tested indicated MIC values smaller or equal to the lowest concentration in the range. Breakpoints of resistance used are indicated with vertical black lines when available.

$\% \mathrm{R}$ - percentage of resistance

$\mathrm{MIC}_{50}, \mathrm{MIC}_{90}$ - the lowest concentration of antimicrobial agent capable of inhibiting the growth of $50 \%$ and $90 \%$ of isolates, respectively. 
Bacterial isolates

Three hundred and thirty two P. multocida isolates collected across the Czech Republic in 2007-2011 were isolated on Columbia blood agar (OXOID, England) plates with 5\% sheep blood overnight at $37{ }^{\circ} \mathrm{C}$. After an additional subculture, cultures $P$. multocida were identified according to standard procedures (Lariviere et al. 1992) and were confirmed by molecular techniques as prescribed previously (Townsend et al. 2001). Eighty three $H$. parasuis isolates were isolated on Columbia blood agar (OXOID) plates with 5\% sheep blood using the "Staph streak" technique and then on chocolate blood agar (OXOID) plates with 5\% sheep blood for NAD growth dependence of $H$. parasuis for $24-48 \mathrm{~h}$ at $37^{\circ} \mathrm{C}$ and were confirmed by a PCR test (Oliveira et al. 2001). All isolates were stored at $-80^{\circ} \mathrm{C}$ in vials containing $0.25 \mathrm{ml}$ Foetal Bovine Serum Gold (PAA Laboratories GmbH, Austria) and $0.25 \mathrm{ml}$ of Cation Adjusted Mueller Hinton Broth II (CAMHB) (Becton, Dickinson and Company, USA).

Antimicrobial susceptibility testing

All $P$. multocida and $H$. parasuis isolates were investigated for their in vitro susceptibilities by the microdilution broth method using custom made microtitre plates (Trek Diagnostic Systems, East England and Trios, Czech Republic). The tested antimicrobial agents and their concentrations of microtitre plates are shown in Table 1. P. multocida isolates were subcultured on Columbia blood agar and $H$. parasuis isolates on Columbia chocolate agar from frozen stock prior to susceptibility testing. Performance and evaluation of the minimal inhibitory concentration (MIC) determination for $P$. multocida followed the recommendations given in document M31-A3 of the CLSI (2008) and the Summary of CLSI Meeting (2011). Currently, there are no CLSIapproved specifications available for the susceptibility testing of $H$. parasuis. Therefore, a modified method of MIC determination and recommended breakpoints for veterinary fastidious microorganisms (Actinobacillus pleuropneumoniae and Histophilus somni) was used according to CLSI (2008). Nevertheless, the interpretive criteria taken from a proposal of clinical breakpoints for amoxicillin (Schwarz et al. 2010) were derived for ampicillin and amoxicillin/clavulanic acid and interpretative breakpoints for tulathromycin (Godinho 2008) were accepted. The susceptibility ranges of $P$. multocida and $H$. parasuis were recorded along with the MIC that inhibited $50 \%\left(\mathrm{MIC}_{50}\right)$ and $90 \%\left(\mathrm{MIC}_{90}\right)$ of the isolates.

Escherichia coli ATCC 2592 and A. pleuropneumoniae ATCC 27090 were used as reference strains for qualitycontrol testing (QC) each bath of the plates and lot of Veterinary Fastidious Medium and cation adjusted Mueller Hinton Broth. QC testing was also performed simultaneously in each series of investigated isolates in permitted ranges.

Statistical analysis

The data were analyzed using the statistical program GraphPad InStat 3. Chi-Squared test was used to determine statistical significance. Differences were considered significant at $P \leq 0.05$.

\section{Results}

The results of susceptibility testing of the $332 \mathrm{P}$. multocida and $83 \mathrm{H}$. parasuis isolates to 12 antimicrobial agents from 2007 to 2011 are shown as a distribution of the MICs, representation of resistant isolates and values of $\mathrm{MIC}_{50}$ and $\mathrm{MIC}_{90}$ in Table 1.

Based on the MIC breakpoint given, the 332 of $P$. multocida isolates showed a low level of resistance to antimicrobial agents, except for tetracycline and tiamulin. The resistance of $P$. multocida isolates to tetracycline was at a high level in $107(32.2 \%)$ isolates. Resistance to tiamulin was at a moderate level in $60(18.1 \%)$ isolates. The resistance to ampicillin, amoxicillin/clavulanic acid, ceftiofur, tulathromycin, tilmicosin, florfenicol and enrofloxacin was at a low level in $20(6.0 \%), 15(4.5 \%), 2$ $(0.6 \%), 8(2.4 \%), 13(3.9 \%), 5(1.5 \%)$ and $5(1.5 \%)$ isolates.

The resistance to antimicrobials of $83 \mathrm{H}$. parasuis isolates was low or not found, except for tetracycline. The resistance of $H$. parasuis isolates to tetracycline was at a high level in $23(27.7 \%)$ isolates. None of $H$. parasuis isolates was resistant to amoxicillin/clavulanic acid, ceftiofur, enrofloxacin and florfenicol. Resistance to tiamulin, gentamicin, tulathromycin, tilmicosin and ampicillin was at a low level in 2 $(2.4 \%), 2(2.4 \%), 3(3.6 \%), 3(3.6 \%)$ and $6(7.2 \%)$ isolates.

The resistance for tiamulin was significantly lower $(P \leq 0.5)$ in $H$. parasuis isolates compared to $P$. multocida. The differences of resistances to other tested antimicrobials were not significant $(P>0.5)$ between $H$. parasuis and $P$. multocida isolates. 


\section{Discussion}

Based on the MIC breakpoint given, $P$. multocida and $H$. parasuis isolates showed in this study a low level of resistance or full susceptibility to antimicrobial agents, with the exception of tetracycline. Furthermore, a moderate level of resistance to tiamulin was found in $P$. multocida isolates. It may be associated with the quantity of antimicrobials used at a certain period, route of administration and dosage, even though in some cases these factors may be insufficient to explain the differences in resistance (Bywater 2004). Tetracyclines are antimicrobials with the highest consumption in veterinary medicine in the Czech Republic. In addition, a significant increase in consumption of antimicrobials was recorded in 2010 for the groups of lincosamides $(+66 \%)$, amfenicols $(+53 \%)$, diterpens $(+31 \%)$ and all generations of cephalosporines $(+21 \%)$. Although the absolute consumption of the tetracycline group decreased slightly from $36,168.68 \mathrm{~kg}$ (in 2009 ) to $35,564.99 \mathrm{~kg}$ (in 2010), analysis of the consumption of antimicrobials reports an increase of therapeutic ingestions due to more frequent administration of doxycycline, which is at lower doses more effective than the formerly more preferred "older" molecules from the tetracycline group (Hera et al. 2011). Higher consumption of amfenicols and cephalosporines at tested $P$. multocida and $H$. parasuis strains did not manifested by an increase in the level of resistance, which remained within the range from undetected to low levels. Lincosamides were not tested in this study, but even though cross-resistance is known among macrolide, triamilide and lincosamide (Kadlec et al. 2011), the level of resistance to tested triamilide (tulathromycin) and macrolide (tilmicosin) remained at a low level.

Following the recommendation that the same methodology and interpretive criteria which can be reanalysed by others if interpretive criteria change (Schwarz et al. 2010), two recent studies of susceptibility of $P$. multocida to antimicrobial agents from Europe were suitable for comparison with our data. In the first study (Lizarazo et al. 2006), in vitro susceptibility of 20 antimicrobial agents was determined for 132 P. multocida isolates collected in Spain during 2003-2004. Using equivalent breakpoint of resistance for tetracycline and tiamulin (CLSI 2008) for swine P. multocida isolates, the percentage of resistant Spanish isolates to tetracycline $(65.9 \%)$ and tiamulin $(50.0 \%)$ were much higher than those of tetracycline and tiamulin in our study.

The second study (Kaspar et al. 2007) described in vitro susceptibility to 24 antimicrobial agents in 471 P. multocida isolates collected from pigs in Germany between 2005 and 2006. Using current breakpoint, there was similarity of the percentage of resistant isolates to ampicillin. In contrast, the resistance of $P$. multocida to amoxicillin/clavulanic acid, ceftiofur, enrofloxacin and florfenicol were not detected, percentage of isolates resistant to tetracycline varied from low level resistance $(7.1 \%$ in breeding pigs) to high level resistance $(21.8 \%$ in piglets). In addition, the percentage of isolates resistant to tiamulin was at a higher level among isolates investigated in all categories of pigs in German study compared to those detected in the $P$. multocida isolates in our study.

All the Czech $H$. parasuis isolates were fully susceptible to $\beta$-lactam antimicrobials with the exception of ampicillin. The same situation was found in Denmark, where $H$. parasuis isolates were fully susceptible to $\beta$-lactams (Aarestrup et al. 2008). Using breakpoints for amoxicillin (Schwarz et al. 2010), high level and very high level of resistance to ampicillin have been found in the United Kingdom $(21.1 \%)$ and Spain $(66.7 \%)$, respectively after reevaluation of published results by De la Fuente et al. (2007). Similarly, higher resistance to enrofloxacin in $H$. parasuis isolates (UK 5.3\%, Spain 36.7\%) was re-evaluated using the proposed breakpoint for gram-negative microorganisms (CLSI 2011).

According to available data, florfenicol exhibited an excellent activity against $H$. parasuis isolates, regardless of their geographic origin (De la Fuente et al. 2007; Aarestrup et al. 2008). Therefore, florfenicol remains useful for the treatment of $H$. parasuis infections. 


\section{Acknowledgements}

This work was supported by the Ministry of Agriculture of the Czech Republic (grant numbers QH 71051, QH 71053 and MZE 0002716202) and the Ministry of Education, Youth and Sports of the Czech Republic (grant number ED0006/01/01 AdmireVet).

\section{References}

Aarestrup F, Duran CO, Burch GS 2008: Antimicrobial resistance in swine production. Anim Health Res Rev 9: 135-148

Bywater RJ 2004: Veterinary use of antimicrobials and emergence of resistance in zoonotic and sentinel bacteria in the EU. J Vet Med B 51: 361-363

Clinical and Laboratory Standards Institute (CLSI) 2008: Performance standards for antimicrobial disk and dilution susceptibility tests for bacteria isolated from animals; informational supplement. NCCLS document M31-A3, vol. 28, No 8. Clinical and Laboratory Standards Institute, Wayne 2008: 99 pp.

Clinical and Laboratory Standard Institute (CLSI), 2011: Summary minutes subcommittee on veterinary antimicrobial susceptibility testing. Clinical and Laboratory Standards Institute. Lake Buena Vista, Florida. 6-7 January 2011. Available at: http://www.clsi.org/Content/NavigationMenu/Committees/Microbiology/ StandingSubcommitteeonVeterinaryAntimicrobialSusceptibilityTesting/MeetingMinutes/VetJanuary2011. pdf. Accessed April 17, 2012.

De la Fuente AJ, Tucker AW, Navas J, Blanco M, Morfia SJ, Gutiérrez-Martín CB 2007: Antimicrobial susceptibility patterns of Haemophilus parasuis from pigs in the United Kingdom and Spain. Vet Microbiol 120: $184-191$

Godinho KS 2008: Susceptibility testing of tulathromycin: Interpretative breakpoints and susceptibility of field isolates. Vet Microbiol 129: 426-432

Hera A, Koutecka L, Dorn D, Pokludova L 2011: Consumption of antibiotics and antiparazitics in veterinary medicine in the Czech Republic between 2003-2010. Available at http://www.uskvbl.cz/attachments/547_1\%20 ATB\%20_spotreba\%202003-2010\%20.doc. Accessed April 17, 2011.

Kadlec K, Brener Michael G, Sweeny MT, Brzuszkiewicz E, Liesegang H, Daniel R, Watts JL, Schwarz S 2011: Molecular basis of macrolide, triamilida and lincosamide resistance in Pasteurella multocida from bovine respiratory disease. Antimicrob Agents Ch 55: 2475-2477

Kaspar H, Schroer U, Wallmann J 2007: Quantitative resistance level (MIC) of Pasteurella multocida isolated from pigs between 2004 and 2006: National resistance monitoring by the BVL. Berlin Munch Tierarztl 120: 442-451

Kolar M, Bardon J, Hanulik V, Sauer P, Babak V, Schlegelova J 2010: Resistance to methicillin in coagulasenegative Staphylococci and its determination. Acta Vet Brno 79: 261-267

Lariviere S, Leblanc L, Mittal KR, Martineau GP 1992: Characterization of Pasteurella multocida from nasal cavities of piglets from farms with or without athropic rhinitis. J Clin Microbiol 30: 1398-1401

Lizarazo YAV, Ferri EFR, De la Fuente AJM, Martin CBG 2006: Evaluation of changes in antimicrobial susceptibility patterns of Pasteurella multocida subsp multocida isolates from pigs in Spain in 1987-1988 and 2003-2004. Am J Vet Res 67: 663-668

Oliveira S, Galina L, Pijoan C 2001: Development of a PCR test to diagnose Haemophilus parasuis infections. J Vet Diagn Invest 13: 495-501

Oliveira S, Pijoan C 2004: Haemophilus parasuis: new trends on diagnosis, epidemiology and control. Review. Vet Microbiol 99: 1-12

Schwarz S, Chaslus-Dancla E 2001: Use of antimicrobials in veterinary medicine and mechanisms of resistance. Vet Res 32: 201-225

Schwarz S, Silley P, Simjee S, Woodford N, van Duijkeren E, Johnson AP, Gaastra W 2010: Assessing the antimicrobial susceptibility of bacteria obtained from animals. Vet Microbiol 141: 1-4

Shin SJ, Kang SG, Nabin R, Kang ML, Yoo HS 2005: Evaluation of the antimicrobial activity of florfenicol against bacteria isolated from bovine and porcine respiratory disease. Vet Microbiol 106: 73 - 77

Townsend KM, Boyce JD, Chung JY, Frost AJ, Adler B, 2001: Genetic organisation of Pasteurella multocida cap Loci and development of a multiplex capsular PCR typing system. J Clin Microbiol 39: 924-929 